\title{
A Biomechanical Comparison Shows No Difference Between Two Knee Braces used for Medial Collateral Ligament Injuries
}

\author{
Joseph M. Gentile, M.D., Michael C. O’Brien, M.A., Bryan Conrad, Ph.D., \\ MaryBeth Horodyski, Ed.D., Michelle L. Bruner, M.S., and Kevin W. Farmer, M.D.
}

\begin{abstract}
Purpose: The purpose of this study was to assess the ability of 2 commonly used knee braces to control knee valgus motion and subsequent strain on the medial collateral ligament (MCL) in a laboratory-controlled environment. Methods: Twenty healthy individuals (6 male, 14 female; mean age, $23 \pm 3$ years) with no history of knee injury or brace use performed a jump landing task while wearing either no brace or 1 of 2 braces: the Playmaker and Total Range of Motion . Three-dimensional joint kinematics and kinetics were measured in our biomechanics laboratory. Results: Significantly less knee dynamic valgus angulation was noted when using either brace $\left(-0.51^{\circ} \pm 3.9^{\circ}\right.$ and $\left.-1.3^{\circ} \pm 3.2^{\circ}\right)$ compared no brace $\left(4.8^{\circ} \pm 3.0^{\circ}\right)$. Dynamic valgus angulation did not differ significantly between the 2 braces tested, which were both not statistically different from baseline alignment. There were significant differences seen in peak knee flexion angle between each brace $\left(77.9^{\circ} \pm 8.8^{\circ}\right.$ and $\left.83.1^{\circ} \pm 8.4^{\circ}\right)$, as well as between both braces and no brace $\left(90.6^{\circ} \pm 11.1^{\circ}\right)$. There was no significant difference in knee frontal plane moment or peak vertical ground reaction force loading among all 3 testing conditions. Conclusions: Compared to no brace, both braces allowed significantly less dynamic valgus angulation of the knee under physiological vertical loads but were not significantly different from one another. Clinical Relevance: Knee braces are commonly used to protect the MCL when placed under physiological loads. It is important to know which braces effectively reduce valgus stress to provide the best outcomes.
\end{abstract}

$\mathbf{T}$ he medial collateral ligament (MCL) of the knee is one of the most commonly injured ligaments in athletes playing sports where their knees are subjected to valgus forces. MCL sprains were the third most common injury behind muscle strains and ankle sprains in professional European soccer players from 2001

From the University of Florida College of Medicine, Department of Orthopedics and Rehabilitation, UF Orthopaedics and Sports Medicine Institute, Gainesville, Florida, U.S.A.

The authors report the following potential conflicts of interest or sources of funding: M.L.B. receives research support from Exactech, Inc.; B.C. is employed with Nike; K.W.F. is a consultant for Arthrex and Exactech, Inc., MB.H. is employed by the National Athletic Trainers' Association as ECE Chair and receives grants from Orthopaedic Research and Education Foundation and Patient Centered Outcomes Research Institute and speaker's fees from Louisiana State University. Full ICMJE author disclosure forms are available for this article online, as supplementary material.

Received May 6, 2020; accepted March 5, 2021.

Address correspondence to Michael C. O'Brien, M.A., UF Orthopaedics and Sports Medicine Institute, 3450 Hull Road, Gainesville, FL 32607, U.S.A. E-mail:michaelcobrien@ufl.edu

(C) 2021 THE AUTHORS. Published by Elsevier Inc. on behalf of the Arthroscopy Association of North America. This is an open access article under the CC BY-NC-ND license (http://creativecommons.org/licenses/by-nc-nd/4.0/).

2666-061X/20792

https://doi.org/10.1016/j.asmr.2021.03.004 through 2008, with 23 days being the average time lost playing because of MCL injury..$^{1-3}$ A study tracking injuries for 1 season in 8 Division I collegiate men's hockey teams found that only concussions occurred more frequently than MCL injuries. ${ }^{4}$ Football and skiing also have a high incidence of MCL sprains. ${ }^{5-9}$ Approximately one quarter of all MCL injuries are isolated, with MCL injuries frequently occurring with patellar instability events or collateral ligament injuries. ${ }^{2,3}$ Anterior cruciate ligament injuries, especially in females, are more commonly seen in sports that involve vertical landing/loading, but MCL injuries are also common in both basketball and volleyball. ${ }^{10,11}$

The majority of MCL injuries respond well to nonoperative treatment, although notable exceptions include chronic injury, multiligamentous injury, and acute grade 3 tibial sided tears. ${ }^{12,13}$ After an MCL injury, early motion with a knee brace is frequently started for protective and rehabilitative purposes. ${ }^{14}$ Although various braces are available to manage rehabilitation from an MCL injury, simple hinged knee braces are most frequently prescribed. Two commonly used braces at our institution are the Playmaker (DonJoy, Dallas, TX) (Fig 1) and the Total Range of Motion (TROM) (DonJoy) (Fig 2). 


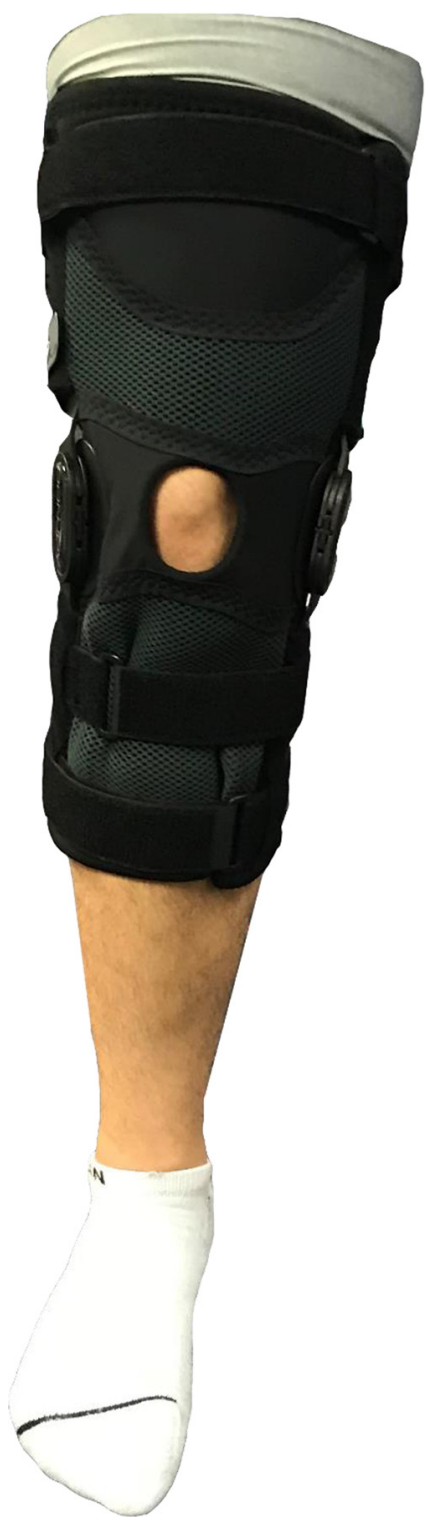

Fig 1. DonJoy Playmaker knee brace.

Whereas more constrained (longer, stiffer) braces are expected to provide more varus/valgus support, they are more cumbersome and not appropriate for return to sport. Previous studies have focused on assessing brace use for prophylactic versus rehabilitative purposes. ${ }^{15,16}$ Biomechanical investigations have studied cadaver models ${ }^{17-21}$ and, more recently, knee "surrogate" models ${ }^{20,22,23}$ that use synthetic materials to simulate the knee. Additionally, some in vivo studies have reported the effect of braces in response to valgus impact ${ }^{23}$ or valgus load ${ }^{24}$ in controlled laboratory settings.

However, it is unknown how effective commonly used knee braces are in protecting the MCL when placed under physiological loads. Understanding how each brace effectively reduces valgus stress will allow physicians to better tailor braces to patients' preferences during the initial rehabilitation phase and subsequent recreational or sporting activities. Furthermore, as more biomechanical studies are completed that investigate the strengths and weakness of commonly used knee braces, the sports rehabilitation community will continue to gain insight on biomechanical indicators of injury risk, patient preference, and cost-effectiveness at varying levels of brace function, performance, and restraint. Our study compared the dynamic valgus angulation that occurred during the landing phase of a drop vertical jump when our subjects were wearing 1 of 2 knee braces or no knee brace.

The purpose of this study was to assess the ability of 2 commonly used knee braces to control knee valgus motion and subsequent strain on the MCL in a laboratory-controlled environment. We hypothesized that either brace would allow less valgus angulation than no brace and that the larger brace (TROM) would allow less dynamic valgus angulation than the smaller brace (Playmaker) during jump landing.

\section{Methods}

\section{Study Design}

We performed a within-subjects repeated-measures study in a controlled laboratory setting. Each subject completed a jump 3 times under 3 conditions (Playmaker brace, TROM brace, and no brace) for a $3 \times 3$ study design. The dependent variables of interest were peak knee valgus angle at landing, peak knee flexion at landing, knee frontal plane moment at landing, and peak vertical ground reaction force.

\section{Power Analysis}

A power analysis was performed to determine sample size. Using a knee frontal plane angle (degrees) reduction of $25 \%$ during valgus strain ${ }^{16}$ and an approximate $7^{\circ}$ change in frontal angle, ${ }^{25}$ we determined that a sample size of 16 patients was necessary to achieve power of $80 \%$ at an $\alpha$ of 0.05 .

\section{Subjects}

This study was approved by the local Institutional Review Board, and all subjects gave informed consent. A total of 20 participants ( 6 male, 14 female; mean age $23 \pm 3$ years) were enrolled. Each subject completed all testing in one session. Participants were recruited from the university community using flyers, posting on our departmental webpage, and word of mouth. We excluded subjects with a history of knee injury or any previous brace use.

\section{Measurements}

Joint kinematics were measured using a 12-camera motion analysis system (Motion Analysis Corp, Santa Rose, CA), with images sampled at $200 \mathrm{~Hz}$. Ground 


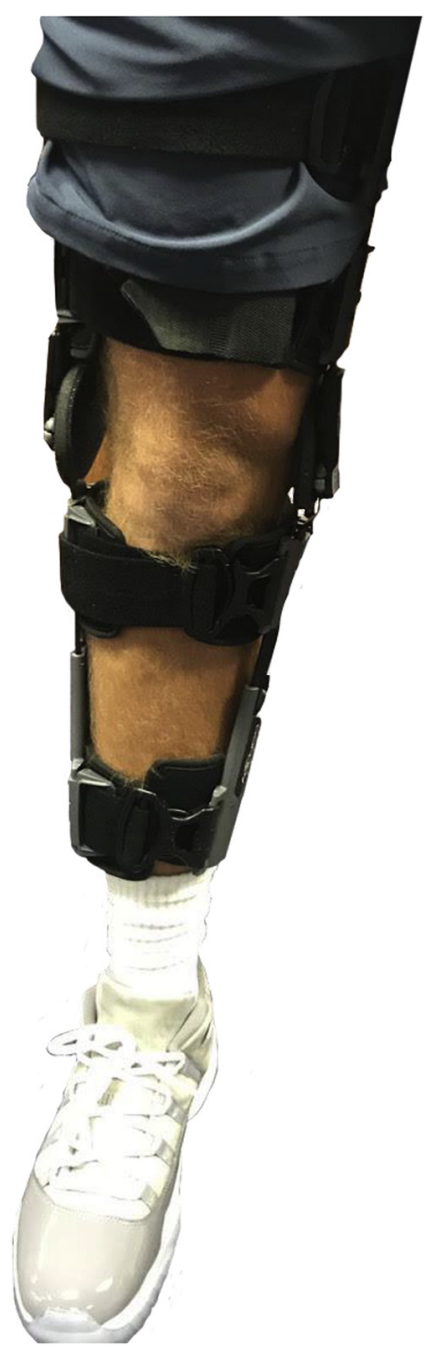

Fig 2. DonJoy Total Range of Motion (TROM) knee brace.

reaction force was measured with 2 AMTI force platforms (AMTI, Inc, Watertown, MA), sampled at 1200 Hz. Data was processed in Visual 3D (C-Motion Inc. Germantown, MD) to calculate joint angles, forces, and moments of the knee during the first landing phase of the drop vertical jump maneuver. Frontal (coronal) plane angles were reported as positive for valgus and negative for varus. For this study, the relevant motion that would cause stress on the MCL injured knee is valgus angulation of the injured knee at landing. As seen in Figure 3, dynamic valgus is the motion of the distal femur toward and motion of the distal tibia away from the midline of the body. This consists of femoral/hip adduction, knee abduction, and ankle eversion.

\section{Study Procedures}

Leg dominance was determined by asking the subject which leg they would use to kick a ball. The dominant leg was the leg used to kick a ball, and the nondominant leg was the stance leg. The brace was placed on the participant's stance leg because it has been shown to be more prone to injury. ${ }^{3,26}$ All participants were fitted for the 2 braces, following the manufacturer's (DonJoy) instructions, by a single experienced researcher. After fitting of the braces, 23 reflective markers were placed on the participant's body (Fig 4) at specific anatomic landmarks with double-sided tape, which allowed the participants' movements to be tracked. These locations include the sacrum and bilateral shoulders, anterior superior iliac spine, greater trochanter, anterior thigh, medial and lateral epicondyles of the knee, tibial tuberosity, medial and lateral malleoli, heel and medial and lateral forefoot. An initial static trial was recorded to build an anatomic model and acclimate participants with their knee brace. We used the drop vertical jump test protocol from a previously published $\operatorname{article}^{27}$ to evaluate jump-landing mechanics. This task has been shown to be sensitive at identifying landing mechanics that are associated with joint injury. ${ }^{28}$

All participants received the same standardized set of instructions and had their questions clarified about the exact jump task to be performed. The subject stood with feet $35 \mathrm{~cm}$ apart on a box $31 \mathrm{~cm}$ off the ground. The subject then dropped directly off the box onto 2 force platforms ( 1 foot on each plate) and immediately performed a maximal vertical leap with arms overhead. A successful test required the subject to look straight forward and both feet to land entirely within the force platforms for both the initial jump and after the

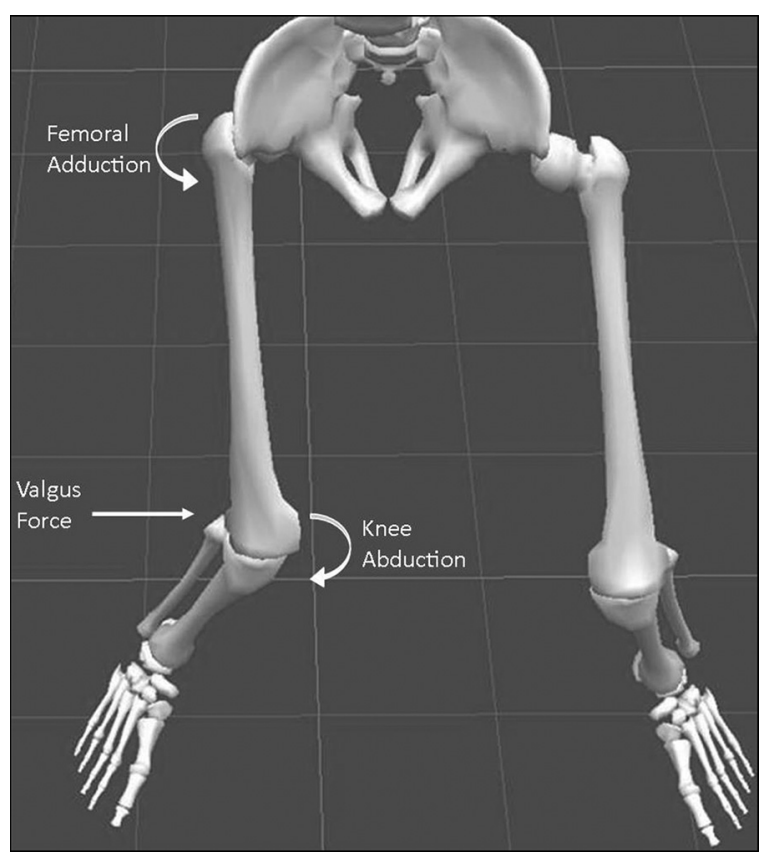

Fig 3. Dynamic valgus was defined as the position or motion, measured in 3 dimensions, of the distal femur toward and distal tibia away from the midline of the body. 


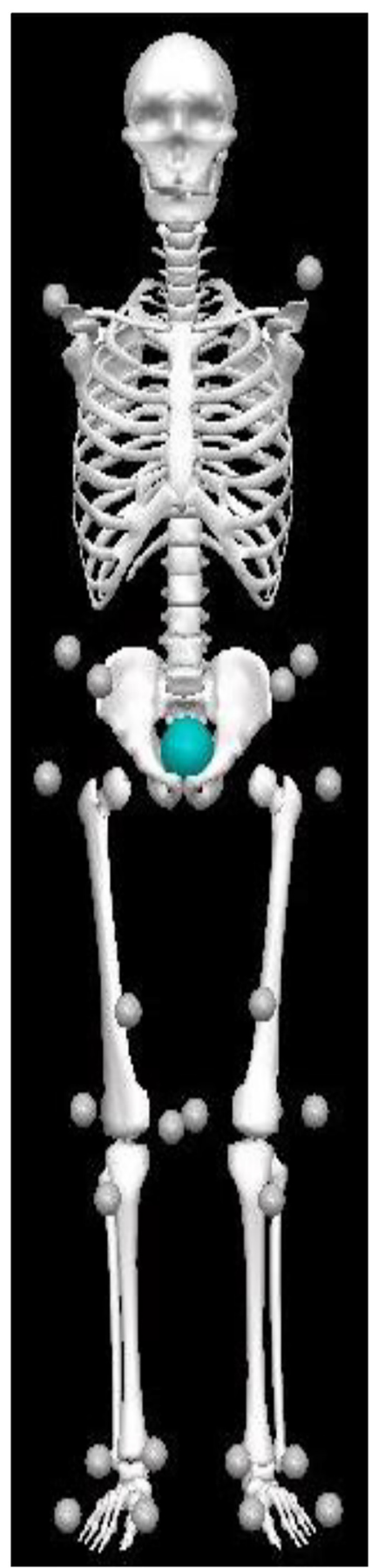

Fig 4. Visual representation of external reflective markers for biomechanical camera tracking. Markers were placed at the sacrum and bilateral shoulders, anterior superior iliac spine, greater trochanter, anterior thigh, medial and lateral epicondyles of the knee, tibial tuberosity, medial and lateral malleoli, heel and medial and lateral forefoot.

maximal leap. Each subject completed the drop vertical jump test 3 times for each condition: Playmaker brace, TROM brace, and no brace. The order of the test conditions was randomized with a computerized number generator.

\section{Statistical Analysis}

Study data was collected and stored using Research Electronic Data Capture (REDCap) system. Statistical analyses were conducted using SPSS version 20.0 (Chicago, IL). Descriptive statistics (means and standard deviations for continuous variables, frequencies and percent for categorical variables) were calculated on study variables and demographics. Statistical analyses included within subjects repeated measures analyses of variance to determine the effect of brace wear on jump landing biomechanics including peak valgus angulation, peak knee flexion, knee frontal plane moment and peak vertical ground reaction force loading. When appropriate a Tukey test was used to delineate pairwise comparisons between the conditions. The level of significance was set at $P<.05$ a priori.

\section{Results}

Our results are illustrated in Table 1 . We found a significant difference $(P<.0001)$ in knee frontal (coronal) plane angle at peak knee flexion between each brace condition and the no brace condition, as both brace conditions allowed significantly less knee valgus angulation compared to the no brace condition. There was no difference in knee frontal plane angle at peak knee flexion between the TROM and Playmaker braces. There was a significant difference $(P<.0001)$ in knee flexion on the braced limb among all 3 brace conditions. There was no significant difference in knee frontal plane moment $(P=.81)$ or peak vertical ground reaction force loading $(P=.22)$ between tested conditions.

There was no significant difference $(P>.05)$ found between males and females' knee frontal plane angle (degrees), peak knee flexion (degrees), knee frontal plane moment $(\mathrm{Nm} / \mathrm{Kg})$, or peak vertical ground reaction force $(\mathrm{N} / \mathrm{Kg})$ across any of the three testing conditions. Male and female knee frontal plane angle (degrees) was reported in Table 2.

\section{Discussion}

Our study found that compared to the no brace condition, both the Playmaker and TROM braces decreased peak valgus angulation of the knee. Vertical ground reaction forces and valgus knee torque loads were not significantly different among the brace conditions. These results did not support our hypothesis that the TROM brace would allow less valgus knee angulation than the smaller Playmaker brace. However, our hypothesis that either brace would allow less valgus knee angulation than no brace was supported. Since females have higher Q-angles and increased propensity to dynamic valgus, ${ }^{27-29}$ we would not have been surprised to see an interaction between gender and kinematics, but our data showed no significant difference in valgus stress. However, concluding that there is no 
Table 1. Biomechanical Motion Analysis Results

\begin{tabular}{lccc}
\hline & No Brace & Playmaker & \multicolumn{1}{c}{ TROM } \\
\hline Knee frontal plane angle & $4.8 \pm 3.0$ & $-0.51 \pm 3.9^{*}$ & $-1.3 \pm 3.2^{*}$ \\
Peak knee flexion & $77.9 \pm 8.8$ & $83.1 \pm 8.4^{*}$ & $90.6 \pm 11.1^{\dagger}$ \\
Knee frontal plane moment $(\mathrm{Nm} / \mathrm{Kg})$ & $0.75 \pm 0.91$ & $0.45 \pm 0.17$ & $0.52 \pm 0.24$ \\
Peak vertical ground reaction force $(\mathrm{N} / \mathrm{Kg})$ & $2.06 \pm 0.41$ & $2.14 \pm 0.69$ & $2.13 \pm 0.32$
\end{tabular}

Both braces allowed significantly less knee valgus angulation than no brace. Frontal plane angles reported as positive for valgus and negative for varus.

*Significant difference $(P<.0001)$ between brace and no brace.

${ }^{\dagger}$ Significant difference $(P<.0001)$ between the Playmaker and TROM braces.

difference between male and female bio mechanical motion from our study is inappropriate as these findings came from a post-hoc analysis of a small dataset that was not designed to adequately address gender differences. Further studies are needed to ascertain a potential bio mechanical difference between males and females

Intuitively, we expected participants to prefer smaller and less constrained braces. However, when queried, the majority of our study participants subjectively stated that the Playmaker felt more constricting and allowed less movement compared to the TROM. An interesting and somewhat unexpected finding was that as the magnitude of constraint increased (no brace < Playmaker < TROM), peak knee flexion increased. Our results differed from the findings of Rishiraj et al., ${ }^{30}$ who showed that acceleration, agility, leg power, and speed were all lower in male athletes wearing a customfitted functional knee brace. These differences, however, disappeared after 14 hours of brace use, suggesting acclimatization. Our subjects did not have an acclimatization period for brace wear. Another reason could be muscle inhibition because of the brace wear. Styf ${ }^{31}$ found that when knee braces compress the soft tissues of the leg, they abnormally elevate intramuscular pressures and decrease local blood flow and muscular oxygenation, which leads to premature muscle fatigue. Osternig and Robertson ${ }^{32}$ found significant differences in both joint (hip, knee, and ankle) position, as well as electromyographic activity between braced and nonbraced subjects from which they concluded neuromuscular control is altered when

Table 2. Male Versus Female Comparison of Frontal Plane Angle (Valgus force)

\begin{tabular}{lccc}
\hline & No Brace & Playmaker & TROM \\
\hline Male & $6.5 \pm 1.4$ & $-2.3 \pm 4.1$ & $-1.7 \pm 1.9$ \\
Female & $4.1 \pm 3.4$ & $0.28 \pm 3.8$ & $-1.1 \pm 3.7$ \\
Total & $4.8 \pm 3.1$ & $-0.51 \pm 4.0$ & $-1.3 \pm 3.2$ \\
$P$ value (male vs. female) & 0.10 & 0.18 & 0.74 \\
\hline
\end{tabular}

Frontal plane angles reported as positive for valgus and negative for varus.

TROM, Total Range of Motion brace. external devices are applied to the lower extremity. Similarly, Wojtys et al. ${ }^{33}$ found slowing in voluntary muscle reaction times in both the hamstrings and quadriceps with brace wear. One or more of these reasons could have contributed to our finding of increased peak knee flexion with increased constraint. However, if performance returns to normal levels after an acclimatization period as suggested by Rishiraj et al., ${ }^{30}$ the effect of soft tissue compression and subsequent muscle fatigue, as well as alterations in neuromuscular control, are either negligible or compensated for over time.

Our study has multiple strengths. We used a wellpublished biomechanical model that simulated physiological vertical loading similar to that generated in actual sport and rehabilitation activities. The use of healthy participants, compared to cadaver or surrogate models, provided a realistic knee motion response to the bracing conditions during sport movement. Additionally, subjects served as their own control because this was a within-subject study. The randomized testing order minimized brace condition bias.

Future research should focus on biomechanical studies involving additional sports-specific movements such as cutting and pivoting. This would allow determination of loads seen by the knee both in and out of these braces and guide decision-making regarding brace wear for sports involving those activities. Future studies could also include a larger sampling of other common knee braces used for management of MCL strains. Last, the sports rehabilitation community might be interested in biomechanical data among specific patient cohorts such as patients with varying levels of athleticism or a history of injured versus uninjured MCLs.

\section{Limitations}

Our study is limited by a small sample size, potentially limiting our ability to find additional differences that may exist between study groups. We limited our study to healthy participants only to investigate performance of 2 braces under ideal conditions. However, there may exist a difference in brace performance and function in individuals with varying ranges of MCL injury. For this 
study, we chose to include both male and female participants, recognizing that anatomic and injury epidemiological differences exist between sexes. Variations in placement of the reflective markers for each subject could lead to differences in measurements between brace conditions. We placed markers on the medial and lateral knee (or brace) so that anatomic coordinate systems could be constructed to minimize sensitivity to these changes in marker location between trials and minimize this risk.

These findings may aid physicians when selecting a knee brace for patients with MCL injuries who are undergoing rehabilitation. Prior cadaver studies demonstrated the effectiveness of reducing dynamic forces on the knee with brace usage. Our in vivo study adds to this existing research by demonstrating that both braces tested provide adequate support to decrease dynamic valgus angulation and subsequent stress on the MCL. Because neither brace was significantly superior to the other, either brace can be used based on the preferences of the physician and patient. At our institution, we now prefer the Playmaker brace, because it allows the transition to return to sport without acquiring a different brace (Level 3 evidence according to the Strength of Recommendation Taxonomy).

\section{Conclusions}

Compared to no brace, both braces allowed significantly less dynamic valgus angulation of the knee under physiological vertical loads but were not significantly different from one another.

\section{References}

1. Ekstrand J, Hägglund M, Waldén M. Injury incidence and injury patterns in professional football: the UEFA injury study. Br J Sports Med 201 1;45:553-558.

2. Lundblad M, Waldén M, Magnusson H, Karlsson J, Ekstrand J. The UEFA Injury Study: 11-year data concerning 346 MCL injuries and time to return to play. Br J Sports Med 2013;47:759-762.

3. Roach CJ, Haley CA, Cameron KL, Pallis M, Svoboda SJ, Owens BD. The epidemiology of medial collateral ligament sprains in young athletes. Am J Sports Med 2014;42: 1103-1109.

4. Flik K, Lyman S, Marx RG. American collegiate men's ice hockey: An analysis of injuries. Am J Sports Med 2005;33: 183-189.

5. Canale ST, Cantler ED, Sisk TD, Freeman BL. A chronicle of injuries of an American intercollegiate football team. Am J Sports Med 1981;9:384-389.

6. Derscheid GL, Garrick JG. Medial collateral ligament injuries in football: Nonoperative management of grade I and grade II sprains. Am J Sports Med 1981;9:365-368.

7. Grace TG, Skipper BJ, Newberry JC, Nelson MA, Sweetser ER, Rothman ML. Prophylactic knee braces and injury to the lower extremity. JBJS 1988;70:422-427.
8. Kim S, Endres NK, Johnson RJ, Ettlinger CF, Shealy JE. Snowboarding injuries: Trends over time and comparisons with alpine skiing injuries. Am J Sports Med 2012;40: 770-776.

9. Meyers MC, Laurent CM, Higgins RW, Skelly WA. Downhill ski injuries in children and adolescents. Sports Med 2007:37:485-499.

10. Iwamoto J, Takeda T, Sato Y, Matsumoto H. Retrospective case evaluation of gender differences in sports injuries in a Japanese sports medicine clinic. Gend Med 2008;5: 405-414.

11. DeHaven KE, Lintner DM. Athletic injuries: Comparison by age, sport, and gender. Am J Sports Med 1986;14: 218-224.

12. Indelicato P. Non-operative treatment of complete tears of the medial collateral ligament of the knee. J Bone Jt Surg 1983;65:323-329.

13. Encinas-Ullán CA, Rodríguez-Merchán EC. Isolated medial collateral ligament tears: An update on management. EFORT Open Rev 2018;3:398-407.

14. Smyth M, Koh J. A review of surgical and nonsurgical outcomes of medial knee injuries. Sports Med Arthrosc Rev 2015;23:e15-22.

15. Sitler M, Ryan CJ, Hopkinson LW, et al. The efficacy of a prophylactic knee brace to reduce knee injuries in football: A prospective, randomized study at West Point. Am J Sports Med 1990;18:310-315.

16. Najibi S, Albright JP. The use of knee braces, Part 1: Prophylactic knee braces in contact sports. Am J Sports Med 2005;33:602-611.

17. Baker BE, VanHanswyk E, Bogosian S, Werner FW, Murphy D. A biomechanical study of the static stabilizing effect of knee braces on medial stability. Am J Sports Med 1987;15:566-570.

18. Baker BE, V E, Orthotist C, et al. The effect of knee braces on lateral impact loading of the knee. Am J Sports Med 1989;17:182-186.

19. Erickson AR, Yasuda K, Beynnon B, Johnson R, Pope M. An in vitro dynamic evaluation of prophylactic knee braces during lateral impact loading. Am J Sports Med 1993;21:26-35.

20. France EP, Paulos LE. In vitro assessment of prophylactic knee brace function. Clin Sports Med 1990;9:823-841.

21. Paulos LE, France EP, Rosenberg TD, Jayaraman G, Abbott PJ, Jaen J. The biomechanics of lateral knee bracing: Part I: Response of the valgus restraints to loading. Am J Sports Med 1987;15:419-429.

22. Cawley PW, France EP, Paulos LE. Comparison of rehabilitative knee braces. A biomechanical investigation. Am J Sports Med 1989;17:141-146.

23. France EP, Paulos LE, Jayaraman G, Rosenberg TD. The biomechanics of lateral knee bracing: Part II: Impact response of the braced knee. Am J Sports Med 1987;15: 430-438.

24. Salvaterra GF, Wang M, Morehouse CA, Buckley WE. An in vitro biomechanical study of the static stabilizing effect of lateral prophylactic knee bracing on medial stability. J Athl Train 1993;28:113-119.

25. Herrington L, Munro A. Drop jump landing knee valgus angle; normative data in a physically active population. Phys Ther Sport 2010;11:56-59. 
26. Krajnc Z, Vogrin M, Rečnik G, Crnjac A, Drobnič M, Antolič V. Increased risk of knee injuries and osteoarthritis in the non-dominant leg of former professional football players. Wien Klin Wochenschr 2010;122:40-43.

27. Ford KR, Myer GD, Hewett TE. Valgus knee motion during landing in high school female and male basketball players. Med Sci Sports Exerc 2003;35:1745-1750.

28. Hewett TE, Myer GD, Ford KR, et al. Biomechanical measures of neuromuscular control and valgus loading of the knee predict anterior cruciate ligament injury risk in female athletes: A prospective study. Am J Sports Med 2005;33:492-501.

29. Chappell JD, Yu B, Kirkendall DT, Garrett WE. A comparison of knee kinetics between male and female recreational athletes in stop-jump tasks. Am J Sports Med 2002;30:261-267.

30. Rishiraj N, Taunton JE, Lloyd-Smith R, Regan W, Niven B, Woollard R. Effect of functional knee brace use on acceleration, agility, leg power and speed performance in healthy athletes. Br J Sports Med 201 1;45:1230-1237.

31. Styf J. The effects of functional knee bracing on muscle function and performance. Sports Med 1999;28:77-81.

32. Osternig LR, Robertson RN. Effects of prophylactic knee bracing on lower extremity joint position and muscle activation during running. Am J Sports Med 1993;21:733-737.

33. Wojtys EM, Kothari SU, Huston LJ. Anterior cruciate ligament functional brace use in sports. Am J Sports Med 1996;24:539-546. 